\title{
Are the current chronic allograft nephropathy grading systems sufficient to predict renal allograft survival?
}

\author{
G.T. Moscoso-Solorzano ${ }^{1,2}$, G. Mastroianni-Kirsztajn ${ }^{1}$, K.S. Ozaki ${ }^{1}$, S. Araujo ${ }^{3}$, \\ M.F. Franco ${ }^{3}$, A. Pacheco-Silva ${ }^{1}$ and N.O.S. Camara ${ }^{1,4}$ \\ ${ }^{1}$ Laboratório de Imunologia Clínica e Experimental, Disciplina de Nefrologia, Universidade Federal de \\ São Paulo, São Paulo, SP, Brasil \\ 2Servicio de Nefrología, Hospital Universitário Central de Asturias, Oviedo, Spain \\ 3Departamento de Patologia, Universidade Federal de São Paulo, São Paulo, SP, Brasil \\ ${ }^{4}$ Laboratório de Imunobiologia de Transplantes, Departamento de Imunologia, Instituto de Ciências \\ Biomédicas IV, Universidade de São Paulo, São Paulo, SP, Brasil
}

Correspondence to: N.O.S. Camara, Disciplina de Nefrologia, UNIFESP, Rua Botucatu, 740, 04023-900

São Paulo, SP, Brasil

Fax: +55+11-5573-9652. E-mail: niels@nefro.epm.br

\begin{abstract}
A major problem in renal transplantation is identifying a grading system that can predict long-term graft survival. The present study determined the extent to which the two existing grading systems (Banff 97 and chronic allograft damage index, CADI) correlate with each other and with graft loss. A total of 161 transplant patient biopsies with chronic allograft nephropathy (CAN) were studied. The samples were coded and evaluated blindly by two pathologists using the two grading systems. Logistic regression analyses were used to evaluate the best predictor index for renal allograft loss. Patients with higher Banff 97 and CADI scores had higher rates of graft loss. Moreover, these measures also correlated with worse renal function and higher proteinuria levels at the time of CAN diagnosis. Logistic regression analyses showed that the use of angiotensin-converting enzyme inhibitor (ACEI), hepatitis $\mathrm{C}$ virus (HCV), tubular atrophy, and the use of mycophenolate mofetil (MMF) were associated with graft loss in the CADI, while the use of $\mathrm{ACEI}, \mathrm{HCV}$, moderate interstitial fibrosis and tubular atrophy and the use of MMF were associated in the Banff 97 index. Although Banff 97 and CADI analyze different parameters in different renal compartments, only some isolated parameters correlated with graft loss. This suggests that we need to review the CAN grading systems in order to devise a system that includes all parameters able to predict long-term graft survival, including chronic glomerulopathy, glomerular sclerosis, vascular changes, and severity of chronic interstitial fibrosis and tubular atrophy.
\end{abstract}

Key words: Renin angiotensin-converting enzyme inhibitor; Chronic allograft damage index; Banff 97; Renal allograft survival; Kidney transplantation

Research supported by CNPq (\#300324/2004-9 and \#302011/2007-2) and by Fundação Oswaldo Ramos. Publication supported by FAPESP.

Received December 19, 2007. Accepted August 26, 2008

\section{Introduction}

Chronic allograft nephropathy (CAN) is present in 40$60 \%$ of allograft biopsies 24 months post-transplantation, and represents the most common cause of late renal graft failure $(1,2)$. CAN is characterized by progressive deterio- ration of renal function produced by sclerotic changes affecting blood vessels, glomeruli, interstitium, and tubules. However, it is graded only by the degree of damage to the last two parameters, interstitium and tubules (3).

Although the Banff 97 classification (4) has become an international standard for evaluating renal allograft biop- 
sies and the presence of chronic lesions with correlation with allograft outcome (5-9), there is another classification used to evaluate renal allograft biopsies: the chronic allograft damage index (CADI) (10). Recently, CADI was validated in a multicenter study as a surrogate end point for long-term graft function (11). Currently, either Banff 97 or CADI classifications is used to quantify renal allograft damage because they are both useful for predicting renal graft outcome $(5,9,11,12)$.

The objective of this study was to determine which of these classifications is the best grading system for predicting loss of graft function due to chronic allograft nephropathy. Furthermore, we also evaluated the independent factors associated with graft loss within both grading systems.

\section{Subjects and Methods}

A total of 1990 consecutive biopsies of deceased and living-donor kidney transplant patients performed at the Federal University of São Paulo (UNIFESP) between 2000 and 2003, with at least 6 months of graft function, were reviewed retrospectively. A total of 450 biopsies with a proven diagnosis of CAN was initially identified. Biopsies were excluded from the study if the patients 1) were recipients of a previous organ transplant, 2) were currently receiving a multiorgan transplant, 3) had a high immunological risk at the time of transplantation, defined as having a previously measured panel-reactive antibody grade of $>60 \%, 4$ ) had de novo post-transplant glomerulonephritis or post-transplantation glomerulonephritis without known primary etiology of chronic renal disease, or 5) had tumors after transplantation. Finally, we included and re-reviewed 161 transplant patient biopsies with chronic allograft nephropathy in this cohort. The Ethics Committee on Human Research of our Institution (UNIFESP) reviewed and approved this study.

\section{Operational definition}

Delayed graft function was defined as the requirement for dialysis during the first week after transplantation without rejection and/or technical problems. Acute rejection was defined as when patients with graft dysfunction presented a biopsy with matched Banff 97 criteria or when the dysfunction resolved after a minimum of 3 doses of methylprednisone over 3 days in the absence of other causes of dysfunction. Any rejection before the 3rd month of transplantation was classified as early acute rejection, while rejection after this period was considered late rejection. Systemic arterial hypertension was defined as repeated elevated blood pressure exceeding 140/90 mmHg or when patients were using at least one anti-hypertensive drug. New onset of diabetes after transplantation (NODAT) was defined as when fasting plasma glucose was $\geq 126$ $\mathrm{mg} / \mathrm{dL}$ ( $\geq 7 \mathrm{mmol} / \mathrm{L}$ ), with random blood sugar level $\geq 200$ $\mathrm{mg} / \mathrm{dL}$ ( $\geq 11.1 \mathrm{mmol} / \mathrm{L}$ ) accompanied by symptoms, or oral glucose tolerance test $\geq 200 \mathrm{mg} / \mathrm{dL}$. Renal function was measured by serum creatinine and by creatinine clearance and calculated using the Cockroft-Gault equation.

\section{Histological analyses}

At least four stained slides were used for quantification of histological changes in each biopsy, one stained with hematoxylin-eosin, one with Masson's trichrome, one with periodic acid-Schiff and one with silver stain. All sections had more than 5 glomeruli per slide. All biopsy specimens were reviewed by two independent pathologists, using both grading systems on each biopsy, before inclusion in the study.

Biopsy specimens of all cases were evaluated and graded according to the Banff 97 criteria (8) and CADI $(10,11)$. CAN was subdivided into three grades according to the Banff 97 classification that evaluates and/or grades the severity of chronic allograft nephropathy based on the evaluation of tubular and interstitial parameters to minimize sampling error problems (4). Grade I (mild) is characterized by mild interstitial fibrosis, grade II (moderate) corresponds to moderate interstitial fibrosis and tubular atrophy, and grade III (severe) corresponds to severe interstitial fibrosis and tubular atrophy and tubular loss.

The CADI score is based on individual component scores for a) diffuse or focal inflammation, b) fibrosis in the interstitium, c) increase in mesangial matrix, d) sclerosis in glomeruli, e) intimal proliferation, and f) tubular atrophy. Each individual parameter is scored from 0 to 3 as described in the literature $(10,11)$. To determine the predictive value of the CADI score for graft loss, we divided the patients into three groups: those with CADI less than 2, those with CADI between 2 and 3.9, and those with CADI equal to or greater than 4, as described by Yilmaz et al. (11).

The kappa index was used to assess inter-rater reliability when observing or coding qualitative/categorical variables (kappa $>0.70$ was considered satisfactory). The kappa index was $=0.85(95 \% \mathrm{Cl}$ : 0.75-0.95), and observed agreement $=0.90$. The result of the severity of acute and chronic lesions in each renal compartment was calculated by applying concordance criteria among these observers. In the event of discrepancy between the two pathologists, the mean value of the variables was used as the final grade of lesion severity.

\section{Statistical analyses}

Pre-transplant demographic characteristics used for covariate-adjusted analyses included dialysis therapy (he- 
modialysis vs peritoneal dialysis), source of the graft (deceased vs living donor), anti-HCV antibodies and antihepatitis B virus serology. The post-transplant related variables included the presence of delayed graft function, early and late acute rejection, cytomegalovirus antigenemia, NODAT, use of angiotensin-converting enzyme inhibitor (ACEI), and use of mycophenolate mofetil (MMF). The presence of proteinuria was recorded as a numerical variable in grams per day and serum creatinine and creatinine clearance were measured at the time of biopsy as well as at follow-up. Chi-square and Fisher exact tests were performed to compare demographic covariates between groups, when appropriate. The Spearman correlation coefficient $\left(r_{s}\right)$ was used to study the relationship between ordinal data or non-normally distributed quantitative data (quantitative clinical variables and histological parameters). One-way ANOVA (and subsequently Bonferroni post hoc test) was used to compare variables among three different Banff 97 and CADI grades.

Data are reported as mean \pm standard deviation and as median and range, when appropriate. A P value of less than 0.05 was considered to be significant. A multivariate, logistic regression model was used to analyze the relationship between the graft loss and the other covariates, including those that were significant in univariate analysis or those variables we considered to be clinically relevant, including immunosuppressive drugs (the use of MMF after CAN diagnosis) and the time of transplantation. Two logistic regression models were built, one model included Banff 97 (also categorized as above grade 2) classification and its histological parameters while another included CADI (also categorized as above 2.3) and its histological parameters. A logistic regression model (backward step) was used to obtain the final model of significant predictors; confounding variables were analyzed when required. There was no confounding when the exponential coefficient (HR) did not change more than $10 \%$. Statistical software SPSS 12.0 (USA) was used for all statistical analyses.

\section{Results}

Demographic analyses of the enrolled population

The study population was divided into two groups: with graft loss $(N=29)$ and without graft loss $(N=132)$. Demographic characteristics and immunosuppressive treatment (intention to treat) are summarized in Table 1. The time of transplant was $57 \pm 29$ months (range 6-130 months) and the mean follow-up after CAN diagnosis was $29 \pm 20$ months (range 10-56 months). During this period, 29 grafts were lost at $58 \pm 34$ months (range 15-134 months) after grafting (Table 1).
Considering the stratification, the patients who lost grafts had more HCV- or HBV-positive serologies ( $P=$ $0.011)$, higher serum creatinine at 12 months post-transplant $(P=0.039)$, and used less ACEI $(P=0.000$, Table 1$)$. Subsequently, we examined the groups according to Banff 97 and CADI grading together with renal function at the time of the graft biopsy. As expected, patients with higher Banff 97 and CADI scores presented higher rates of graft lost, higher proteinuria and serum creatinine, although creatinine clearance as assessed by Cockroft-Gault formula was similar (Table 2).

To better investigate this relationship, we re-analyzed these data taking into consideration the three grades of Banff 97 and three scores of CADI. Once more, there was a statistically significant trend toward worse renal function in those patients with higher grades (Banff 97 grade III and CADI >4). The age of transplant at biopsy was higher in Banff 97 grade III and CADI >4. Patients with Banff 97 grade I and CADI $<2$ lost fewer grafts (9 and 7\%, respectively) than the other groups (Table 3 ).

\section{Relationship between CADI and Banff 97 scores and their correlation with renal function}

The CADI correlated with tubular atrophy $\left(r_{s}=0.655, P\right.$ $=0.000)$, interstitial fibrosis $\left(r_{s}=0.657, P=0.000\right)$, vascular fibrosis intimal thickening $\left(r_{s}=0.497, P=0.000\right)$ and with mesangial matrix increase $\left(r_{s}=0.609, P=0.000\right)$. The Banff 97 grade correlated with glomerular sclerosis $\left(r_{\mathrm{s}}=\right.$ $0.216, P=0.006)$, interstitial fibrosis $\left(r_{s}=0.664, P=0.000\right)$ and tubular atrophy $\left(r_{s}=0.651, P=0.000\right)$. There was also correlation between the CADI histological parameters and Banff 97 scores as reported in Table 4.

The CADI and Banff 97 scores correlated with serum creatinine and creatinine clearance at the time of biopsy. Interestingly, 24-h proteinuria at the time of biopsy only correlated with CADI; however, the correlation was weak ( $r$ $=0.296, P=0.01$ ). Serum creatinine or creatinine clearance levels at $0,6,12,24,36,48$, and 60 months after transplantation did not correlate with any histological parameter (data not shown).

\section{Multivariate analyses}

Two models were employed for multivariate analyses using logistic regression: one with Banff 97 parameters and the other with CADI parameters. With the first model, we observed that the use of $A C E I(H R=0.309, P=0.011)$, positive hepatitis $C$ serology $(H R=3.788, P=0.010)$, the use of MMF (HR = 0.304, $\mathrm{P}=0.007)$, and moderate interstitial fibrosis and tubular atrophy (grade $\geq 2 ; \mathrm{HR}=$ $2.946, P=0.014$ ) were associated with graft loss. Similarly, in the model that included the CADI and its histological 
Table 1. Demographic data.

\begin{tabular}{|c|c|c|}
\hline Variables & No graft loss $(N=132)$ & Graft loss $(\mathrm{N}=29)$ \\
\hline Recipient age (years) & $34(9-65)$ & $31(14-54)$ \\
\hline Donor age (years) & $40(6-66)$ & $36(4-59)$ \\
\hline Gender (male) & $85(64.3 \%)$ & $14(48.2 \%)$ \\
\hline Deceased donor & $44(33.3 \%)$ & $12(41.3 \%)$ \\
\hline Time of transplant (months) & $51(6-146)$ & $48(5-134)$ \\
\hline Time of transplant at enrollment (months) & $20(3-138)$ & $25(5-125)^{*}$ \\
\hline Hemodialysis therapy & $123(93.1 \%)$ & $29(100 \%)$ \\
\hline Early acute renal rejection & $54(40.9 \%)$ & $13(44.8 \%)$ \\
\hline Late acute renal rejection & $16(12.1 \%)$ & $8(27.5 \%)^{*}$ \\
\hline Steroid-resistant rejection & $10(7.5 \%)$ & $4(13.7 \%)$ \\
\hline Delayed graft function & $44(33.3 \%)$ & $11(37.9 \%)$ \\
\hline Diabetes mellitus post-transplantation & $15(11.3 \%)$ & $4(13.7 \%)$ \\
\hline Anti-CMV antibodies (IgG) & $22(16.7 \%)$ & $6(20.6 \%)$ \\
\hline Anti-HCV & $17(12.8 \%)$ & $10(34.4 \%)^{*}$ \\
\hline Serum creatinine $(\mathrm{mg} / \mathrm{dL})$ at 7 th day post-transplant & $1.60(0.60-6.50)$ & $1.40(0.80-7.80)^{\star}$ \\
\hline Serum creatinine $(\mathrm{mg} / \mathrm{dL})$ at 12 th month post-transplant & $1.60(0.60-3.50)$ & $1.70(0.90-6.20)^{*}$ \\
\hline Proteinuria $(\mathrm{g} / 24 \mathrm{~h})$ at 12 th month post-transplant & $0.19(0.00-5.10)$ & $0.11(0.00-1.10)$ \\
\hline ACEI use & $71(53.7)$ & $10(34.4)^{\star}$ \\
\hline \multicolumn{3}{|l|}{ Initial immunosuppressive therapy } \\
\hline Cyclosporine & $111(84.0 \%)$ & $26(89.6 \%)$ \\
\hline Tacrolimus & $15(11.3 \%)$ & $3(10.3 \%)$ \\
\hline Rapamycin & $8(6.0 \%)$ & $1(3.4 \%)$ \\
\hline Azathioprine & $94(71.2 \%)$ & $21(72.4 \%)$ \\
\hline Mycophenolate mofetil & $26(19.6 \%)$ & $6(20.6 \%)$ \\
\hline \multicolumn{3}{|l|}{ Final immunosuppressive therapy } \\
\hline Cyclosporine & $103(78.0 \%)$ & $23(79.3 \%)$ \\
\hline Tacrolimus & $14(10.6 \%)$ & $4(13.7 \%)$ \\
\hline Rapamycin & $3(2.2 \%)$ & $2(6.8 \%)$ \\
\hline Azathioprine & $24(18.1 \%)$ & $5(17.2 \%)$ \\
\hline Mycophenolate mofetil & $102(77.2 \%)$ & $17(58.6 \%)^{*}$ \\
\hline
\end{tabular}

Data are reported as median with range in parentheses or as number with percent in parentheses. CMV = cytomegalovirus; $\mathrm{HCV}=$ hepatitis $\mathrm{C}$ virus; $\mathrm{ACEI}=$ angiotensin-converting enzyme inhibitor. ${ }^{*} \mathrm{P}<0.05$ compared to no graft loss ( $t$-test).

Table 2. Renal function and chronic allograft nephropathy (CAN) histology as a function of graft loss.

\begin{tabular}{lcc}
\hline Variables & No graft loss $(\mathrm{N}=132)$ & Graft loss $(\mathrm{N}=29)$ \\
\hline $\begin{array}{l}\text { CAN grade according to Banff } 97 \\
\quad\end{array}$ & $10(34.4 \%)^{*}$ \\
$\quad$ rrade I & $81(61.3 \%)$ & $14(48.2 \%)^{*}$ \\
Grade II & $39(29.5 \%)$ & $5(17.4 \%)^{*}$ \\
Grade III & $12(9.2 \%)$ & $3(10.4 \%)^{*}$ \\
CAN grade according to CADI & & $17(58.6 \%)^{*}$ \\
CADI $<2$ & $62(31.8 \%)$ & $9(31.0 \%)^{*}$ \\
CADI $\geq 2$ to $<4$ & $22(17.5 \%)$ & $3.23 \pm 1.91^{*}$ \\
CADI $\geq 4.0$ & $2.22 \pm 0.92$ & $1.31 \pm 1.61^{*}$ \\
Serum creatinine $(\mathrm{mg} / \mathrm{dL})$ at biopsy & $0.84 \pm 1.27$ & $32 \pm 115$ \\
Proteinuria (g/24 $\mathrm{h})$ at biopsy & $44 \pm 18$ & \\
Creatinine clearance $(\mathrm{mL} / \mathrm{min})$ at biopsy & &
\end{tabular}

Data are reported as number with percent in parentheses or as mean \pm SD. See Subjects and Methods for explanation of Banff $97(8)$ and $\operatorname{CADI}(10,11)$ scoring systems. CADI = chronic allograft damage index. ${ }^{*} \mathrm{P}<0.05$ compared to no graft loss (chi-square, Fisher and $t$-tests). 
parameters, the use of ACEI (HR $=0.286, P=0.009)$, positive hepatitis $C$ serology $(H R=3.027, P=0.037)$, the use of MMF ( $\mathrm{HR}=0.300, \mathrm{P}=0.008)$, and tubular atrophy $(\mathrm{HR}=4.768, \mathrm{P}=0.002)$ were the variables associated with graft loss. On the other hand, the presence of acute rejec- tion, delayed graft function, Banff 97 parameters (chronic glomerulopathy, mesangial matrix increase, tubular atrophy, vascular thickening, and arteriolar hyalinosis), CADI parameters (sclerosis in glomeruli, mesangial matrix, intimal proliferation of vessel, and diffuse or focal inflamma-

Table 3. Renal function and graft loss rate scored according to the Banff 97 and CADI grading systems.

\begin{tabular}{|c|c|c|c|}
\hline Banff 97 & Grade I (N = 91) & Grade II (N = 53) & Grade III ( $\mathrm{N}=17)$ \\
\hline Serum creatinine $(\mathrm{mg} / \mathrm{dL})$ at enrollment & $2.00 \pm 0.57$ & $2.62 \pm 1.12$ & $3.92 \pm 2.33$ \\
\hline Proteinuria $(\mathrm{g} / 24 \mathrm{~h})$ at enrollment & $0.72 \pm 1.03$ & $0.94 \pm 1.31$ & $1.82 \pm 2.2$ \\
\hline Creatinine clearance $(\mathrm{mL} / \mathrm{min})$ at enrollment & $47 \pm 18$ & $37 \pm 15$ & $28 \pm 18$ \\
\hline Graft loss & $8(9 \%)$ & $16(30 \%)$ & $5(30 \%)$ \\
\hline Transplantation time (months) & $53 \pm 25$ & $65 \pm 34$ & $56 \pm 32$ \\
\hline Transplant time at enrollment & $22 \pm 22$ & $39 \pm 32$ & $46 \pm 33$ \\
\hline CADI & CADI $<2(\mathrm{~N}=45)$ & CADI $\geq 2$ and $<4(\mathrm{~N}=85)$ & $\mathrm{CADI} \geq 4(\mathrm{~N}=31)$ \\
\hline Serum creatinine $(\mathrm{mg} / \mathrm{dL})$ at enrollment & $1.89 \pm 0.51$ & $2.36 \pm 0.96$ & $3.27 \pm 1.96$ \\
\hline Proteinuria $(\mathrm{g} / 24 \mathrm{~h})$ at enrollment & $0.51 \pm 0.58$ & $0.76 \pm 1.06$ & $1.82 \pm 2.1$ \\
\hline Creatinine clearance $(\mathrm{mL} / \mathrm{min})$ at enrollment & $50 \pm 19$ & $41 \pm 15$ & $32 \pm 17$ \\
\hline Graft loss & $3(7 \%)$ & $17(20 \%)$ & $9(29 \%)$ \\
\hline Transplantation time (months) & $45 \pm 22$ & $60 \pm 31$ & $65 \pm 32$ \\
\hline Transplant time at enrollment & $15 \pm 15$ & $32 \pm 29$ & $47 \pm 32$ \\
\hline
\end{tabular}

Data are reported as mean \pm SD or as number with percent in parentheses. See Subjects and Methods for explanation of Banff 97 and CADI scoring systems. CADI = chronic allograft damage index. Grades I, II, and III as well as CADI $<1, \mathrm{CADI} \geq 2$ and $\leq 4$ and CADI $>4$ were statistically different for each variable (one-way ANOVA).

Table 4. Correlation between Banff 97 classification and CADI.

\begin{tabular}{|c|c|c|}
\hline Variables & Banff 97 & CADI index \\
\hline CADI & $0.568(P=0.00)$ & 1 \\
\hline Banff 97 classification & 1 & $0.568(P=0.00)$ \\
\hline Serum creatinine $(\mathrm{mg} / \mathrm{dL})$ at biopsy & $0.383(P=0.00)$ & $0.381(P=0.00)$ \\
\hline Proteinuria $(\mathrm{g} / 24 \mathrm{~h})$ at biopsy & $0.169(P=0.05)$ & $0.296(P=0.01)$ \\
\hline Creatinine clearance $(\mathrm{mL} / \mathrm{min})$ at biopsy & $-0.382(P=0.00)$ & $-0.390(P=0.00)$ \\
\hline \multicolumn{3}{|l|}{ Banff 97 scores } \\
\hline Chronic glomerulopathy & $0.270(P=0.01)$ & $0.370(P=0.00)$ \\
\hline Mesangial matrix increase & $0.433(P=0.00)$ & $0.609(P=0.00)$ \\
\hline Tubular atrophy & $0.761(P=0.00)$ & $0.655(P=0.00)$ \\
\hline Interstitial fibrosis & $0.779(P=0.00)$ & $0.657(P=0.00)$ \\
\hline Arteriolar hyaline thickening & $0.337(P=0.00)$ & $0.465(P=0.00)$ \\
\hline Vascular fibrosis intimal thickening & $0.401(P=0.00)$ & $0.497(P=0.00)$ \\
\hline \multicolumn{3}{|l|}{ CADI } \\
\hline Glomerular sclerosis & $0.216(P=0.006)$ & $0.625(P=0.00)$ \\
\hline Mesangial matrix increase & $0.406(P=0.00)$ & $0.663(P=0.00)$ \\
\hline Interstitial fibrosis & $0.664(P=0.00)$ & $0.826(P=0.00)$ \\
\hline Diffuse or focal inflammation & $0.270(P=0.00)$ & $0.670(P=0.00)$ \\
\hline Tubular atrophy & $0.651(P=0.00)$ & $0.838(P=0.00)$ \\
\hline Vascular intimal proliferation & $0.516(P=0.00)$ & $0.659(P=0.00)$ \\
\hline
\end{tabular}

Data are reported as $r_{S}$ and $P$ values calculated by the Spearman test. CADI = chronic allograft damage index. 
Table 5. Multivariate analysis of variables using the CADI classification.

\begin{tabular}{lcccc}
\hline Variables & P & Exp(B) & SE & Wald \\
\hline Initial model & & & & \\
Use of ACEI & 0.036 & 0.310 & 0.559 & 4.382 \\
Acute renal rejection & 0.588 & 1.286 & 0.465 & 0.294 \\
Delayed graft function & 0.958 & 0.975 & 0.473 & 0.03 \\
Hepatitis C virus & 0.031 & 3.502 & 0.581 & 4.650 \\
Transplantation time & 0.242 & 0.990 & 0.08 & 1.370 \\
Use of MMF after CAN & 0.012 & 0.308 & 0.469 & 6.291 \\
CADI (>2.3) & 0.954 & 1.051 & 0.864 & 0.003 \\
Sclerosis in glomeruli - CADI & 0.720 & 0.828 & 0.527 & 0.129 \\
Mesangial matrix - CADI & 0.475 & 1.519 & 0.585 & 0.510 \\
Tubular atrophy - CADI & 0.083 & 15.472 & 1.580 & 3.005 \\
Fibrosis in the interstitium - CADI & 0.554 & 0.441 & 1.383 & 0.350 \\
Intimal proliferation of vessel - CADI & 0.735 & 0.810 & 0.623 & 0.115 \\
Diffuse or focal inflammation - CADI & 0.614 & 0.739 & 0.599 & 0.254 \\
& & & & \\
Final model & & & & \\
Use of ACEI & 0.009 & 0.286 & 0.476 & 6.912 \\
Hepatitis C virus & 0.037 & 3.027 & 0.531 & 4.356 \\
Use of MMF after CAN & 0.008 & 0.300 & 0.453 & 7.043 \\
Tubular atrophy - CADI & 0.002 & 4.768 & 0.502 & 9.667 \\
\hline
\end{tabular}

$\operatorname{Exp}(B)=$ confidence interval for $\operatorname{Exp}(B) ;$ Wald = Wald statistical test; SE = standard error; $\mathrm{ACEI}=$ angiotensin-converting enzyme inhibitor; $\mathrm{MMF}=$ mycophenolate mofetil; $\mathrm{CAN}=$ chronic allograft nephropathy $\mathrm{CADI}=$ chronic allograft damage index.

Table 6. Multivariate analysis of variables using the Banff 97 classification.

\begin{tabular}{lcccc}
\hline Variables & P & Exp(B) & SE & Wald \\
\hline Initial model & & & & \\
Use of ACEI & 0.040 & 0.365 & 0.491 & 4.199 \\
Acute renal rejection & 0.749 & 1.158 & 0.458 & 0.102 \\
Delayed graft function & 0.885 & 0.934 & 0.473 & 0.021 \\
Hepatitis C virus & 0.012 & 4.316 & 0.580 & 6.362 \\
Transplantation time & 0.232 & 0.991 & 0.008 & 1.431 \\
Use of MMF after CAN & 0.007 & 0.277 & 0.477 & 7.242 \\
Banff 97 classification (>grade II) & 0.155 & 3.132 & 0.802 & 2.026 \\
Chronic glomerulopathy (>grade II) & 0.633 & 0.630 & 0.968 & 0.228 \\
Mesangial matrix increase (>grade II) & 0.626 & 1.324 & 0.576 & 0.238 \\
Tubular atrophy (>grade II) & 0.335 & 0.424 & 0.889 & 0.930 \\
Interstitial fibrosis (>grade II) & 0.180 & 3.066 & 0.836 & 1.797 \\
Vascular fibrosis intimal thickening (>grade II) & 0.177 & 0.493 & 0.524 & 1.821 \\
Arteriolar hyaline thickening (>grade II) & 0.123 & 2.121 & 0.488 & 2.376 \\
& & & & \\
Final model & & & & \\
Use of ACEI & 0.011 & 0.309 & 0.463 & 6.449 \\
Hepatitis C virus & 0.010 & 3.788 & 0.519 & 6.585 \\
Use of MMF after CAN & 0.007 & 0.304 & 0.440 & 7.339 \\
Banff 97 classification (>grade II) & 0.014 & 2.946 & 0.440 & 6.030 \\
\hline
\end{tabular}

$\operatorname{Exp}(B)=$ confidence interval for $\operatorname{Exp}(B) ;$ Wald = Wald statistical test; $S E$ = standard error; $\mathrm{ACEI}=$ angiotensin-converting enzyme inhibitor; $\mathrm{MMF}=$ mycophenolate mofetil; $\mathrm{CAN}=$ chronic allograft nephropathy. tion) and the time between transplantation and diagnosis were not statistically significant (Tables 5 and 6).

\section{Discussion}

A major problem in renal transplantation is identifying reliable early morphological changes that can help predict renal allograft outcome. Acute and chronic tissue damage can be semi-quantified by the Banff 97 classification, while CADI provides information about chronic injury. The Banff 97 schema grades the severity of chronic allograft nephropathy based on tubular and interstitial parameters (4). The CADI index is more extensive regarding the number of parameters, also taking into account vascular and glomerular changes, which are evaluated differently. Moreover, the Banff 97 classification does not take into consideration glomerulosclerosis, while CADI cannot evaluate arteriolar hyalinosis and chronic glomerulopathy. Thus, although both systems are used for the same purpose, they actually look at the tissue differently, and possibly make different assumptions for the graft outcome.

The present study investigated whether the Banff 97 and CADI classifications correlated well with each other, even though they evaluate lesions in different renal compartments, and also whether they can both predict graft loss in patients with chronic allograft nephropathy.

The CADI correlated with graft function at the moment of biopsy as did the Banff 97 classification. These findings were consistent with other published studies $(5-7,9,10)$. However, extending these studies, we observed that the systems had some limitations in predicting renal graft loss when they were considered as whole scores. When all histological variables associated with renal function were considered, tubular atrophy and in- 
terstitial fibrosis were identified as independent predictors of renal graft loss.

These data confirmed the findings described by Nankivell et al. (13) in which interstitial fibrosis showed an adverse impact on graft outcome (13-17). Furthermore, interstitial fibrosis is a common finding in other chronic kidney diseases besides chronic allograft nephropathy (13), having strong correlation with tubular atrophy and graft loss $(13,18)$. According to published reports (18-21), chronic interstitial fibrosis can develop after primary tubular injury, resulting in alteration in tubular phenotype with production of chemoattractant for monocyte/macrophages (22). The non-specific mononuclear inflammation around and within atrophied tubules in native kidney may essentially reflect an immune response that may trigger fibrosis, a process to which tubular cells may actively contribute. Conversely, inflammation in the interstitium with capillary injury may lead to ischemia with tubular atrophy and fibrosis. Nankivell and colleagues (23) reported that the same process occurred in renal allograft, whereby the interstitial fibrosis and tubular atrophy scores are increased due to subclinical immune-mediated injury and nephrotoxicity.

Glomerulosclerosis was not a predictor for graft loss, perhaps because, in the present study, there were patients who lost the graft without having significant glomerulosclerosis at the time of biopsy (lower transplantation time could be implicated in less glomeruli lesion), and possibly because those with any suspicion of glomerulopathy were excluded. This result correlates, in part, with the literature $(3,13,15)$, where glomerulosclerosis could result from interstitial fibrosis, with development of periglomerular fibrosis and atubular glomeruli, or from a high degree of arteriolar damage leading to ischemic glomeruli.

In the present study, the use of ACEl therapy had a protective role on graft loss. In many animal models and

\section{References}

1. Joosten SA, Sijpkens YW, van Kooten C, Paul LC. Chronic renal allograft rejection: pathophysiologic considerations. Kidney Int 2005; 68: 1-13.

2. Massy ZA, Guijarro C, Wiederkehr MR, Ma JZ, Kasiske BL. Chronic renal allograft rejection: immunologic and nonimmunologic risk factors. Kidney Int 1996; 49: 518-524.

3. Chapman JR, O'Connell PJ, Nankivell BJ. Chronic renal allograft dysfunction. J Am Soc Nephrol 2005; 16: 30153026.

4. Racusen LC, Solez K, Colvin RB, Bonsib SM, Castro MC, Cavallo T, et al. The Banff 97 working classification of renal allograft pathology. Kidney Int 1999; 55: 713-723.

5. Freese P, Svalander CT, Molne J, Norden G, Nyberg G. some clinical studies (24-27), such protection has already been established. An experimental study by the Remuzzi group (26) showed that ACEI therapy could indeed suspend proteinuria and glomerulosclerosis development, interrupting the progression of chronic allograft dysfunction in rodents.

In the multivariate analyses, some classical variables were not associated with graft loss while others corroborated data from the literature. Moreso and colleagues (28) have already shown that $\mathrm{HCV}$ was an independent predictor of graft survival. Acute rejection is a stronger negative factor on graft survival and its role in the development of graft loss is well established in literature (29). However, in the two models in the present study, we did not observe this association. We assume that although acute rejection was prevalent in both groups, the percentage of steroidresistant episodes was small, minimizing its impact on graft loss.

We observed that ACEI and MMF therapies had a positive impact on graft loss. The reno-protective effect of ACEI has been established in a wide variety of progressive diabetic and non-diabetic renal diseases (27,30,31). In renal transplantation, there are few clinical studies that show the beneficial effects of ACEI $(32,33)$. Since MMF has been introduced in renal transplantation, there are some reports demonstrating its benefit in $\operatorname{CAN}(34,35)$. Ojo et al. (34) in a study with 66,774 human renal allograft recipients showed that MMF reduces late allograft loss independent of its effects on acute rejection.

Our data indicated that Banff 97 and CADI, applied independently, were insufficient to predict renal graft loss. This suggests that the two systems indeed analyze different parameters and should either be combined in order to better predict graft outcome, or the best individual parameter of each grading system should be combined.
Chronic allograft nephropathy - biopsy findings and outcome. Nephrol Dial Transplant 2001; 16: 2401-2406.

6. Seron D, Moreso F, Bover J, Condom E, Gil-Vernet S, Canas C, et al. Early protocol renal allograft biopsies and graft outcome. Kidney Int 1997; 51: 310-316.

7. Nickerson $P$, Jeffery J, Gough J, McKenna R, Grimm P, Cheang $\mathrm{M}$, et al. Identification of clinical and histopathologic risk factors for diminished renal function 2 years posttransplant. J Am Soc Nephrol 1998; 9: 482-487.

8. Grandaliano G, Di Paolo S, Monno R, Stallone G, Ranieri E, Pontrelli $P$, et al. Protease-activated receptor 1 and plasminogen activator inhibitor 1 expression in chronic allograft nephropathy: the role of coagulation and fibrinolysis in renal 
graft fibrosis. Transplantation 2001; 72: 1437-1443.

9. Di Paolo S, Stallone G, Schena A, Infante B, Gesualdo L, Paolo SF. Hypertension is an independent predictor of delayed graft function and worse renal function only in kidneys with chronic pathological lesions. Transplantation 2002; 73: 623-627.

10. Isoniemi H, Taskinen E, Hayry P. Histological chronic allograft damage index accurately predicts chronic renal allograft rejection. Transplantation 1994; 58: 1195-1198.

11. Yilmaz S, Tomlanovich S, Mathew T, Taskinen E, Paavonen $\mathrm{T}$, Navarro M, et al. Protocol core needle biopsy and histologic Chronic Allograft Damage Index (CADI) as surrogate end point for long-term graft survival in multicenter studies. J Am Soc Nephrol 2003; 14: 773-779.

12. Ortiz $F$, Paavonen $T$, Tornroth $T$, Koskinen $P$, Finne $P$, Salmela K, et al. Predictors of renal allograft histologic damage progression. J Am Soc Nephrol 2005; 16: 817-824.

13. Nankivell BJ, Borrows RJ, Fung CL, O'Connell PJ, Allen RD, Chapman JR. Evolution and pathophysiology of renaltransplant glomerulosclerosis. Transplantation 2004; 78: 461-468.

14. Nankivell BJ, Borrows RJ, Fung CL, O'Connell PJ, Chapman $\mathrm{JR}$, Allen RD. Delta analysis of posttransplantation tubulointerstitial damage. Transplantation 2004; 78: 434-441.

15. Hariharan S, McBride MA, Cohen EP. Evolution of endpoints for renal transplant outcome. Am J Transplant 2003; 3: 933-941.

16. Hariharan S, Kasiske B, Matas A, Cohen A, Harmon W, Rabb $\mathrm{H}$. Surrogate markers for long-term renal allograft survival. Am J Transplant 2004; 4: 1179-1183.

17. Halloran PF, Melk A, Barth C. Rethinking chronic allograft nephropathy: the concept of accelerated senescence. J Am Soc Nephrol 1999; 10: 167-181.

18. Grandaliano G, Gesualdo L, Ranieri E, Monno R, Stallone G, Schena FP. Monocyte chemotactic peptide-1 expression and monocyte infiltration in acute renal transplant rejection. Transplantation 1997; 63: 414-420.

19. Pascual M, Theruvath T, Kawai T, Tolkoff-Rubin N, Cosimi $A B$. Strategies to improve long-term outcomes after renal transplantation. N Engl J Med 2002; 346: 580-590.

20. Hotchkiss H, Chu TT, Hancock WW, Schroppel B, Kretzler $\mathrm{M}$, Schmid $\mathrm{H}$, et al. Differential expression of profibrotic and growth factors in chronic allograft nephropathy. Transplantation 2006; 81: 342-349.

21. Nangaku M. Chronic hypoxia and tubulointerstitial injury: a final common pathway to end-stage renal failure. J Am Soc Nephrol 2006; 17: 17-25.

22. Fogo $A B$. Progression and potential regression of glomerulosclerosis. Kidney Int 2001; 59: 804-819.
23. Nankivell BJ, Borrows RJ, Fung CL, O'Connell PJ, Allen $\mathrm{RD}$, Chapman JR. The natural history of chronic allograft nephropathy. N Engl J Med 2003; 349: 2326-2333.

24. Heinze G, Mitterbauer C, Regele H, Kramar R, Winkelmayer WC, Curhan GC, et al. Angiotensin-converting enzyme inhibitor or angiotensin II type 1 receptor antagonist therapy is associated with prolonged patient and graft survival after renal transplantation. J Am Soc Nephrol 2006; 17: 889-899.

25. Remuzzi A, Fassi A, Bertani T, Perico N, Remuzzi G. ACE inhibition induces regression of proteinuria and halts progression of renal damage in a genetic model of progressive nephropathy. Am J Kidney Dis 1999; 34: 626-632.

26. Remuzzi A, Gagliardini E, Donadoni C, Fassi A, Sangalli F, Lepre MS, et al. Effect of angiotensin II antagonism on the regression of kidney disease in the rat. Kidney Int 2002; 62: 885-894.

27. Remuzzi G, Benigni A, Remuzzi A. Mechanisms of progression and regression of renal lesions of chronic nephropathies and diabetes. J Clin Invest 2006; 116: 288-296.

28. Moreso $F$, Ibernon M, Goma M, Carrera M, Fulladosa X, Hueso $M$, et al. Subclinical rejection associated with chronic allograft nephropathy in protocol biopsies as a risk factor for late graft loss. Am J Transplant 2006; 6: 747-752.

29. Hariharan S, Johnson CP, Bresnahan BA, Taranto SE, McIntosh MJ, Stablein D. Improved graft survival after renal transplantation in the United States, 1988 to 1996. N Engl J Med 2000; 342: 605-612.

30. Taal MW, Brenner BM. Renoprotective benefits of RAS inhibition: from ACEI to angiotensin II antagonists. Kidney Int 2000; 57: 1803-1817.

31. Kshirsagar AV, Joy MS, Hogan SL, Falk RJ, Colindres RE. Effect of ACE inhibitors in diabetic and nondiabetic chronic renal disease: a systematic overview of randomized placebo-controlled trials. Am J Kidney Dis 2000; 35: 695-707.

32. Artz MA, Hilbrands LB, Borm G, Assmann KJ, Wetzels JF. Blockade of the renin-angiotensin system increases graft survival in patients with chronic allograft nephropathy. Nephrol Dial Transplant 2004; 19: 2852-2857.

33. Remuzzi G, Perico N. Routine renin-angiotensin system blockade in renal transplantation? Curr Opin Nephrol Hypertens 2002; 11: 1-10.

34. Ojo AO, Meier-Kriesche HU, Hanson JA, Leichtman AB, Cibrik D, Magee JC, et al. Mycophenolate mofetil reduces late renal allograft loss independent of acute rejection. Transplantation 2000; 69: 2405-2409.

35. Merville P, Berge F, Deminiere C, Morel D, Chong G, Durand $D$, et al. Lower incidence of chronic allograft nephropathy at 1 year post-transplantation in patients treated with mycophenolate mofetil. Am J Transplant 2004; 4: 1769-1775. 